\title{
Channel Assignment Mechanism for Multiple APs Cochannel Deployment in High Density WLANs
}

\author{
Jianjun Lei $\mathbb{D}$, Jianhua Jiang, and Fengjun Shang $\mathbb{D}$ \\ School of Computer Science and Technology, Chongqing University of Posts and Telecommunications, Chongqing 400065, China \\ Correspondence should be addressed to Jianjun Lei; leijj@cqupt.edu.cn
}

Received 5 September 2017; Revised 29 December 2017; Accepted 4 January 2018; Published 7 February 2018

Academic Editor: Zhaolong Ning

Copyright (C) 2018 Jianjun Lei et al. This is an open access article distributed under the Creative Commons Attribution License, which permits unrestricted use, distribution, and reproduction in any medium, provided the original work is properly cited.

\begin{abstract}
In Wireless Local Area Networks (WLANs), cochannel deployment can bound channel access delay and improve network capacity due to mitigating the collision and interference among different Access Points (APs). In this paper, we present a network model and an interference model for multiple APs cochannel deployment and propose a channel assignment mechanism which formulates the channel assignment problem into a time slot allocation problem. Meanwhile, we assign the channel based on the vertex coloring algorithm and make extra polls by utilizing the time slot reservation strategy to improve the channel assignment. Furthermore, we optimize the polling list of APs through classifying the clients to improve the channel utilization. The simulation results show that our proposed algorithm can improve the performance in terms of network throughput, transmission delay, and packet loss rate compared with the DCF (Distributed Coordination Function) and TMCA algorithms.
\end{abstract}

\section{Introduction}

In recent years, with the development of wireless technologies, the demand of WLANs has risen and its deployment has become denser and denser. The wireless APs are placed everywhere in our daily life environment $[1,2]$. A plenty of APs will cause serious collision, influencing users' experience. Hence, eliminating interference among APs under the limited number of channels has become an important issue.

Typically, the clients for IEEE 802.11 operate on the DCF mode, in which clients use the carrier sense medium access with collision avoidance (CSMA/CA) mechanism [3] to compete for the wireless medium to transmit data. Due to the contention nature and throughput fair characteristic of DCF, it is difficult to provide guaranteed bandwidth and bounded access time for each client. Especially, in high density and multi-AP WLANs, high collision probability leads to low efficiency of channel access, and thus too much time is spent in dealing with collision for retransmission [4]. This significantly affects networks performance and user's experience. Although many optimal extensions of DCF are proposed, the competition among nodes cannot be eased and the transmission failure and retransmission increase sharply with the increase of the deployment density. Due to the limitation of DCF, some literatures suggest adopting centralized channel access mechanism such as the Point Coordination Function (PCF). Although PCF provides contention-free channel access among clients, it is mainly designed to be used in single-AP network and cannot be directly used in multi-AP WLANs.

In this paper, we propose a multi-AP deployment framework, which lets all APs operate in the same channel and coordinates them to obtain the appropriate channel, reducing the system interference. The core idea of the algorithm is to maintain moderate cooperation among APs by the central Access Controller (AC) to achieve high channel utilization. As a result, transmission collision is reduced and wasted time is minimized. We first formulate the channel assignment problem into vertex coloring problem and then present an improved channel assignment strategy which makes extra poll for APs without collision. It is referred to as CCA (cochannel assignment) mechanism and it has been demonstrated that this strategy can improve the channel utilization and guarantee the bandwidth demand in our previous work [5]. Meanwhile, aiming at the possible empty polling problem, we also propose a priority channel access strategy 
to improve the channel utilization, which is referred to as optimized CCA mechanism. We preserve as much features of IEEE802.11 standard as possible. Thus, little hardware modification for APs is needed.

The contributions of this paper are listed as follows.

(1) We present the system framework of cochannel deployment and workflow of coordination data traffic among APs, which formulates the channel assignment problem into vertex coloring problem and gives the constraint conditions for channel allocation.

(2) Benefiting from the cochannel deployment framework, we propose a basic CCA algorithm and an optimized CCA algorithm, which not only provides the high concurrent data transmission, but also reduces some empty poll to further enhance the channel utilization.

(3) Compared to our previous work, we conduct some more simulations under the different network densities and scales to demonstrate the feasibility and improvement of algorithm.

The rest of this paper is organized as follows: we survey some related works in Section 2; in Section 3, the network interference model and conflict constraint are introduced; the cochannel assignment strategy and the priority channel access strategy are presented in Section 4; we evaluate the performance of the proposed algorithm in Section 5 and make conclusion in Section 6.

\section{Related Work}

In WLANs, to degrade the interference, the researchers usually assign nonoverlapping channels to neighboring APs. Achanta proposes the classic Least Congested Channel Search (LCCS) [6] algorithm. In the algorithm, APs scan each channel and detect the condition of data transmission in all channels. APs can obtain the load level in each channel and choose the channel in which the load is lighter and the interference is smaller. Since this method only assigns the channel statically on the AP side, the performance may drop sharply under the high density environment. In [7], the authors propose a kind of global channel allocation method based on graph theory. The main idea is to select the vertex according to the saturation and adjacent degree of vertices to color from the available color sets. A similar channel assignment scheme is proposed in [8], where the expected transmission delay due to the interference from a neighboring BSS is regarded as the weight of the edge. In [9], a kind of distributed flexible channel assignment, called DFCA, is designed to assign the channel width and center frequency to each link based on the interfering relation among clients in the network. Similarly papers $[10,11]$ maximize throughput by the way of allocating nonoverlapping channel. The authors of paper $[12,13]$ try to eliminate the interference by researching channel allocation and power control jointly. However, both of the methods do not consider the competition between different APs and load distributions. Thus, due to a limited number of channels available, they cannot eliminate interference completely.

Other literatures focus on eliminating the interference by utilizing the PCF (Point Coordination Function). In [14], the authors propose a kind of collision-free client polling method to mitigate the conflict, which polls the clients in a time slot manner. Though the interference is eliminated effectively, this method needs too much time slots to poll all clients so that the utilization of channel is degraded. In [15], a client polling framework, named $\mathrm{MiFi}$, is proposed for multi-AP deployment, in which CFPs (contention-free periods) at all APs are synchronized and divided into time slots. Neighboring APs are assigned with different time slots, so as to poll all clients without collisions. Nevertheless, in the AP interference model, there are too many constraints to suppress the concurrent polls, thus resulting in the decline of aggregated throughput. In [16], the authors describe an adaptive AP cooperation scheme where each AP autonomously controls its polling period according to channel conditions. Yet, these algorithms mainly concentrate on allocating nonoverlapping channels to APs while the coordination mechanism between APs is ignored.

In this paper, we propose a collision-free channel assignment algorithm to improve the aggregated throughput by utilizing cochannel deployment, which consists of three procedures: (1) conducting a basic channel assignment by vertex coloring algorithm that determines the minimum number of time slots; (2) constructing an improved channel assignment by making extra polls for APs to improve the channel utilization; (3) classifying the clients to optimize the polling list of APs and reduce empty polls. Additionally, since all APs only operate in one channel in our algorithm, multiple vertically nonoverlapping channels also can be used to improve the network capacity in high density WLANs.

\section{Network Interference Model and Conflict Constraint}

3.1. Network Model. Figure 1 shows a framework of network deployment. All APs operate in the PCF mode and utilize the same channel. Each client is associated with the AP which has the largest received signal strength indication (RSSI) and can obtain an optimal physical data rate according to the channel condition. The central AC gathers the information of clients by all APs. And with the global information, the AC makes the client data transmission decision in a centralized manner. The workflow is shown in Figure 2.

Due to the global network state awareness of centralized data transmission, the AC gathers all necessary information from other components in this system. In our scheme, the information refers to the associating relation between APs and clients and the neighboring relation among all APs and clients. Then, with the global information, the AC can make an interference table. According to this table, the AC can make an appropriate transmission decision when the AP wants to make polling with a client.

Furthermore, the contention-free periods (CFPs) of APs are divided into several time slots, and within each time slot the AP polls one client or transmits a frame to one of its clients. Different client may have different physical data rates; thus the referred length of time slot $\Delta$ can be calculated roughly by

$$
\Delta=\frac{L_{\mathrm{MaxMPDU}}}{\sum r\left(C_{r} / C\right)},
$$




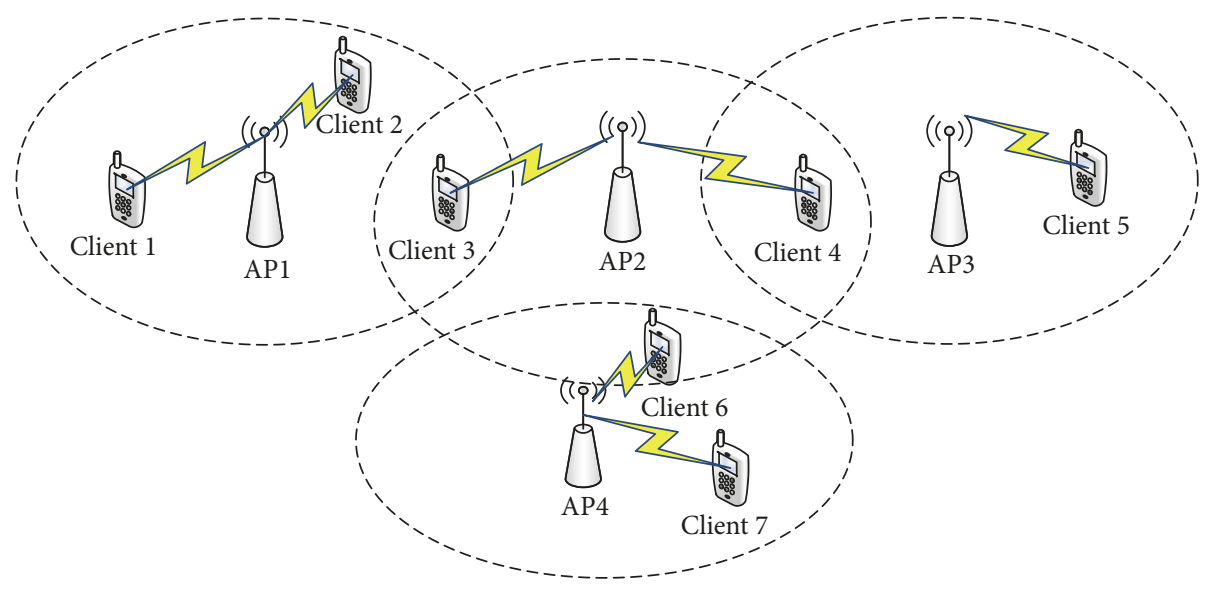

Figure 1: An example of WLAN deployment.

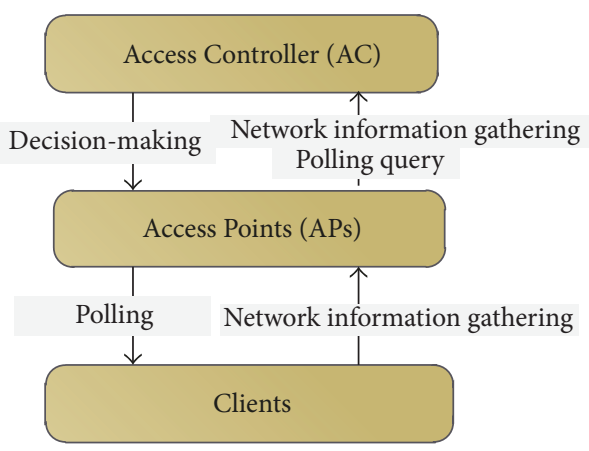

Figure 2: Workflow of clients and APs by centralized AC.

where $L_{\text {MaxMPDU }}$ is the length of the maximum Message Protocol Data Unit (MPDU) and $C$ and $C_{r}$ are the total number of clients and the number of clients whose data rate is $r$, respectively. In addition, the value of $\Delta$ can be updated periodically to adapt to the dynamic of network.

3.2. Interference Model. In our proposed channel assignment mechanism, since all APs are operating in the PCF mode, there would not be any interference between two clients in the same AP. However, two clients from neighboring APs may interfere with each other. Assume that two APs of $\mathrm{AP}_{m}$ and $\mathrm{AP}_{n}$ have $S$ clients totally. The interference probability $I_{e}$ between $\mathrm{AP}_{m}$ and $\mathrm{AP}_{n}$ can be estimated by

$$
I_{e}=\frac{\rho_{1}+\rho_{2}+\cdots+\rho_{s}}{S}
$$

Equation (2) is subject to

$$
\rho_{s}= \begin{cases}1, & \text { if } \exists(i, j) \in \Phi_{m} \times \Phi_{n} \text { s.t. } i, j \text { interfere } \\ 0, & \text { otherwise, }\end{cases}
$$

where $\rho_{s}$ describes the interference relationship and $\Phi_{m}$ and $\Phi_{n}$ are the sets of clients associated with $\mathrm{AP}_{m}$ and $\mathrm{AP}_{n}$, respectively. Note that the threshold $I_{\text {th }}$ can change according to the network scenario.

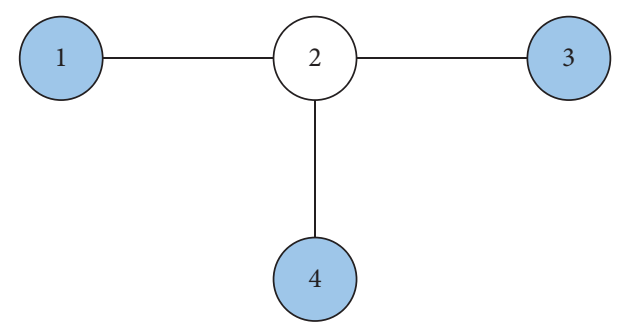

FIGURE 3: Polling conflict graph of the example of WLAN.

3.3. Conflict Constraint. Naturally, only one client in the same AP is allowed to transmit or receive data during a time slot. Simultaneously, to avoid transmission collisions, interfering clients from neighboring APs should be polled in different time slots. Hence, a variable $T_{i}$ is defined to denote the time slot assigned to client $i$ and then such conflict constraint can be formally expressed as follows:

$$
\begin{aligned}
& 1 \leq T_{i} \leq R, \quad \forall i \in R, \\
& T_{i} \neq T_{j}, \quad \text { if } I_{e}=1, \forall i, j \in C, \\
& T_{i} \neq T_{j}, \quad \text { if } A_{m}=A_{n}, \forall i, j \in C, \forall m, n \in A,
\end{aligned}
$$

where $R$ is the number of time slots, $C$ is the set of clients, and $A$ is the set of APs. Furthermore, the conflict constraint of concurrent polling can also be described by a polling conflict graph $G=(V, E)$, where each vertex represents an AP, and there is an edge between two vertexes if the interference probability $I_{e}$ exceeds the threshold $I_{\text {th }}$. Figure 3 shows the polling conflict graph for WLANs deployment in Figure 1.

Note that the problem of polling conflict graph can be solved by the vertex coloring algorithm. In other words, allocating time slot to the corresponding AP in the network is equivalent to coloring a vertex in the graph. However, it is a $K$-colorable problem in graph theory, which is a well-known NP-hard problem. 


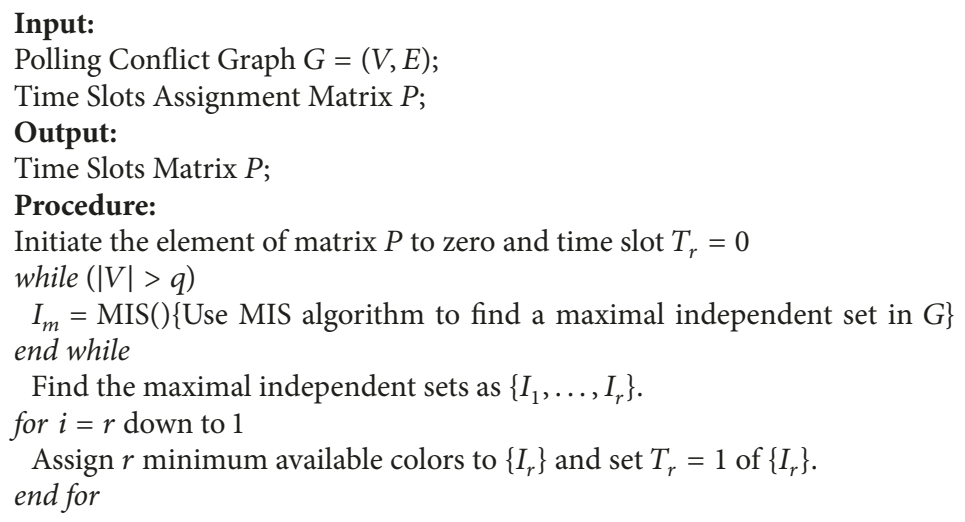

Algorithm 1: The basic channel assignment procedure.

\section{Channel Assignment Algorithm}

In this section, we propose the CCA mechanism based on vertex coloring for cochannel channel assignment, which includes a basic channel assignment and an improved channel assignment strategy. In addition, to minimize polling overhead, we also propose an optimized CCA, which arranges the different channel access priority based on the clients' load level.

4.1. Basic Channel Assignment. The basic channel assignment for a cochannel deployment is aiming to avoid the interference among APs by allocating time slots to all APs in a collision-free manner based on PCF. It includes two components. The first one is the coloring algorithm given a graph $G=(V, E)$ and the number of colors $R$, which seeks out a feasible color scheme with minimal number of colors. The second component works out an efficient slot assignment by using the coloring scheme, which also can be described by time slots assignment matrix.

To solve the vertex coloring problem, a mass of heuristic algorithms has been proposed in graph theory and many of them have been applied into practice, such as the maximal independent set (MIS) [17] and the EXTRACOL [18] which both can provide the optimal results. Thus, we use the MIS algorithm to solve the problem of the vertex coloring graph and realize the basic channel assignment strategy. The procedure is described by Algorithm 1.

We construct the interference collision graph by using the proposed interference model. And then, the MIS algorithm is applied to solve the coloring problem repeatedly and the coloring scheme is recorded. After that, the time slots assignment matrix is established according to the coloring scheme and all APs can poll their associating clients in the assigned time slots. By this procedure, an AP cannot poll any of its associating clients when one of its neighboring APs is receiving or transmitting data from or to its clients.

4.2. Time Slots Reservation Scheme. In the basic channel assignment, part of APs may be idle in some time slots. To utilize such idle time slots adequately and improve channel utilization, we propose an improved channel assignment mechanism that is called time slots reservation (TSR) scheme. Crucially, the AP can poll its associating clients in idle time slots by querying the polling list of all the neighboring APs. As mentioned in previous section, such queries can be supported by the AC with the global network neighboring table and data transmission dynamic information. Therefore, we define the variable $C_{\text {sen }}$ to denote the set of clients which are in the carrier sensing range of APs. The procedure is described by Algorithm 2.

The idle APs in the next time slot query the set $N$ consisting of all adjacent APs and also query the polling list of all neighboring APs in the set $N$. By that, APs can judge whether the next polling client satisfies the conflict constraint, which can avoid the interference among the neighboring APs in the next polling. By this procedure, the concurrent poll is permitted among the neighboring APs, thus resulting in improving the channel utilization. A concurrent data transmitting example shown in Figure 1 is that client 3 can be required to transmit data by the polling from AP2 when the AP1 is transmitting the data to client 1 . However, in legacy IEEE802.11 DCF or PCF standard, this concurrency cannot occur due to their CSMA/CA mechanism.

4.3. Priority Channel Access Strategy. Some undesirable poll occurring will result in the wasting of bandwidth if the AP polls some clients without data to send or receive. Therefore, two-class priority client polling mechanism based on queuing theory is proposed in some literatures [19-21]. However, due to the side-effects of PCF, they work inefficiently in multiple APs cochannel deployment and high density WLANs. Thus, to minimize the waste of bandwidth and polling overhead, we present an optimized CCA strategy, which classifies the clients into three categories and polls them in different priority. The procedure is described by Algorithm 3 .

In this strategy, an extra field is used to describe whether the clients need to transmit data. The clients in the polling list of AP are classified into three categories: (1) the clients with both uplink and downlink data, (2) the clients with uplink or downlink data, and (3) the clients without data needed to 


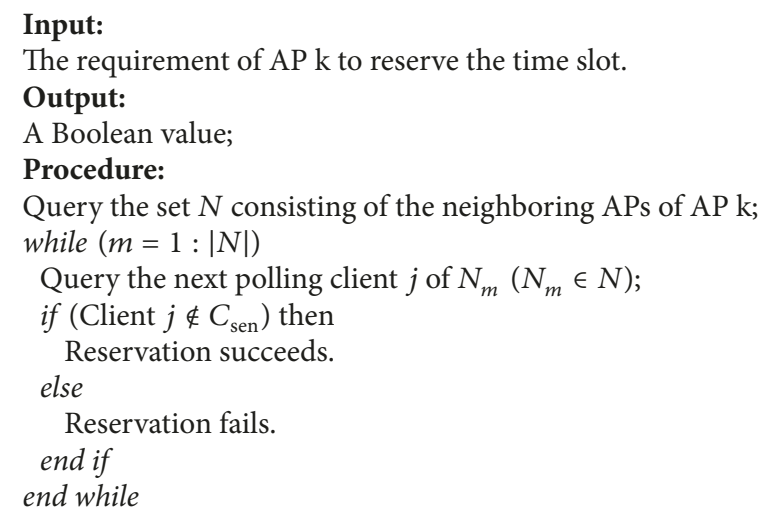

Algorithm 2: The improved channel assignment procedure.

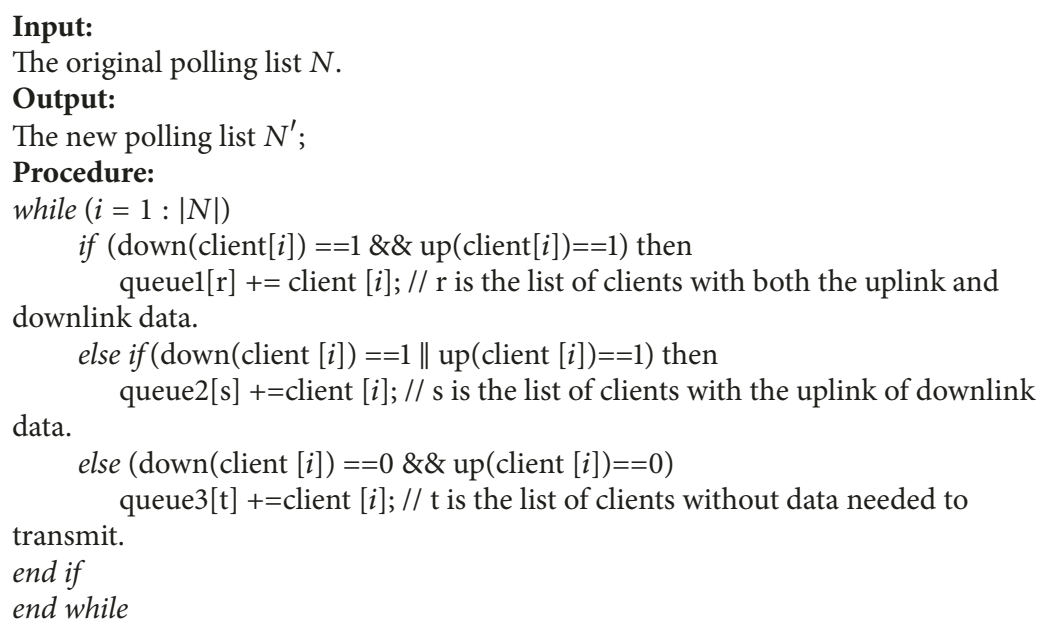

Algorithm 3: Priority channel access strategy.

transmit. The AP polls the clients in category 1 with highest priority, assuming that the probability is $1-P$. And then, the clients in categories 2 and 3 are polled with probability $P$. Note that the value of probability $P$ should be adjusted and the polling list should be updated according to the real network scenario. It is noted that only a trivial modification for the data frame of client is needed to adapt this mechanism. In legacy IEEE802.11 standard, one bit field "more data" in "frame control" field is used to denote that more data will be transmitted from AP to some sleeping clients. In our algorithm, this flag bit also is used by the clients; thus the extra modification hardly occurs for clients. In addition, the time slot allocations for both basic channel assignment and improved channel assignment are updated only when interference or network topology changes. Therefore, the overhead of both mechanisms is negligible compared with data traffic in the network.

\section{Performance Evaluations}

5.1. Simulation Methodology. In this section, we conduct simulations by OPNET14.5, evaluate the proposed CCA and optimized CCA mechanisms, and compare them with the basic DCF method and the TMCA algorithm proposed in [22]. In the simulation, we set up the FTP server to simulate the real network traffic and set the uplink and downlink traffic in half, respectively. The experimental parameters are shown in Table 1 . The clients are randomly distributed in a $1000 \times 800 \mathrm{~m}^{2}$ field and each client is associated with an AP according to the largest RSSI mechanism mentioned in previous section. And we acquire the transmission rate of each client by utilizing the relationship between the transmission rate $r_{i j}$ and the Signal to Interference plus Noise Ratio (SINR) $\gamma_{i j}$, which is shown in Table 2 [23].

We evaluate the performance in terms of transmission delay, packet loss rate, and network throughput under the 


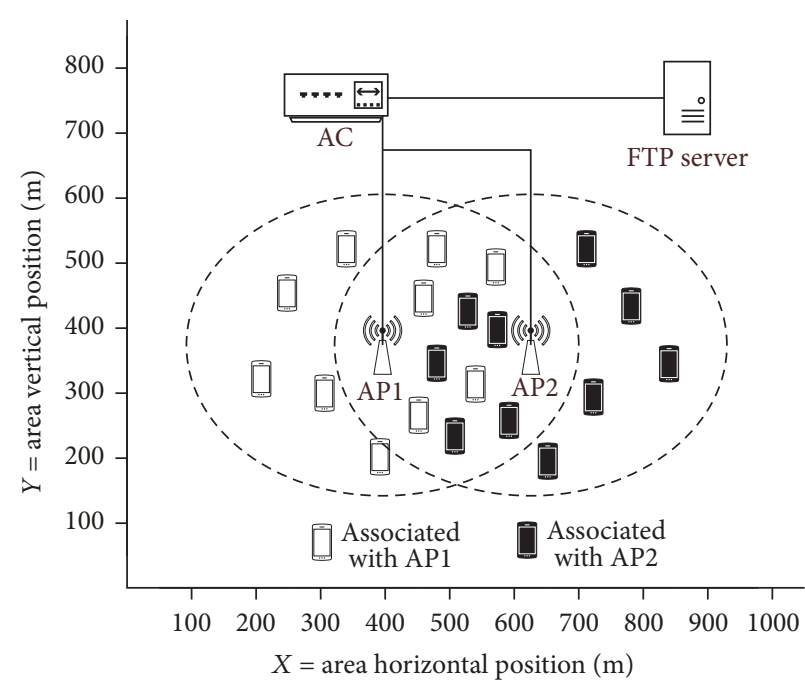

(a) Tight coupling scenario

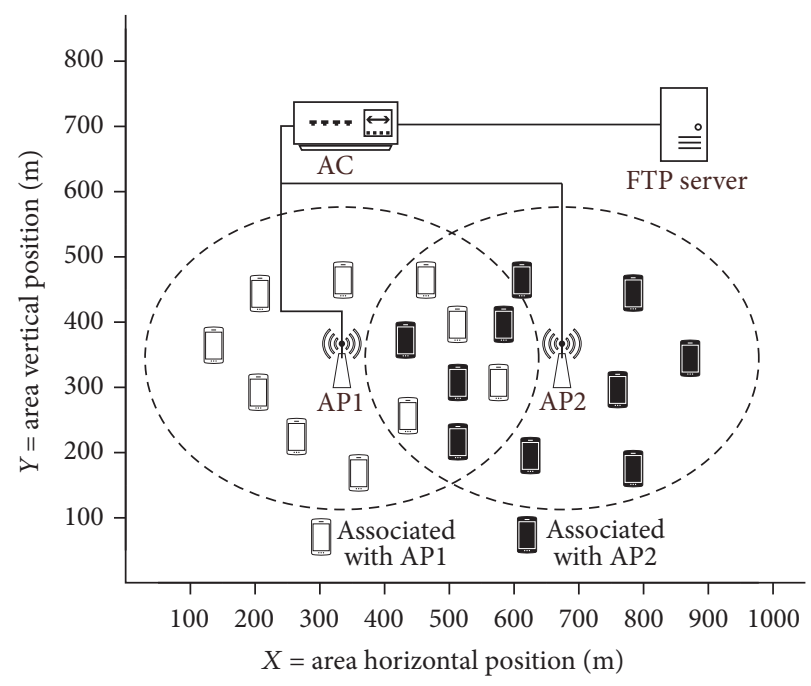

(b) Loose coupling scenario

Figure 4: Basic network deployment with two APs.

TABLE 1: Experimental parameters.

\begin{tabular}{lc}
\hline Parameters & Value \\
\hline ACK & 14 bytes \\
RTS & 20 bytes \\
DIFS & 50 us \\
CTS & 14 bytes \\
SIFS & 10 us \\
EIFS & 300 us \\
\hline
\end{tabular}

scenario of basic and high density network deployment. The transmission delay refers to the average time from the sending time in source client to the reception time in target client, which is computed by

$$
T=\frac{1}{N} \sum_{i=1}^{N}(\mathrm{RT}(i)-\mathrm{ST}(i)),
$$

where $N$ is the number of successfully transmitted data frames and $\mathrm{RT}(i)$ and $\mathrm{ST}(i)$ are the reception and transmission times of the $i$ th data frame, respectively.

5.2. Simulation in Basic Network Deployment. The basic network deployment is shown in Figure 4. Two APs are deployed in the scenario of tight coupling and loose coupling, which denotes that the APs have the same coverage overlap area but they can interfere with each other directly or indirectly, respectively.

5.2.1. Transmission Delay. We first examine the transmission delay of the DCF, CCA, and optimized CCA algorithms under various client densities, where the number of clients increases from 5 to 30 for each AP. As shown in Figure 5, the transmission delay of three algorithms increases with the increasing of the number of clients. Reasonably, a larger number of clients result in higher collision probability. Nevertheless, our proposed CCA and optimized CCA algorithms perform almost similarly and better compared with the DCF scheme in both tight and loose coupling deployment. The delay of our algorithms is reduced by $45 \%$ in tight coupling and $32 \%$ in loose coupling, respectively.

5.2.2. Packet Loss Rate. We also evaluate the packet loss rate under various client densities. Figure 6 shows the simulation results of three algorithms. Obviously, our proposed algorithms can achieve slightly lower packet loss rate in both tight and loose coupling deployment. The reason is that our algorithms utilize the time-sharing scheduling strategy, which can reduce the occurrence conflict and thus result in the lower packet loss rate.

5.2.3. Network Throughput. Figure 7 shows the average network throughput tendency as the network density changes. When the number of clients for each AP is not beyond 20, the DCF algorithm obtains better throughput compared with the CCA and optimized CCA algorithms, which may be due to little channel collision and low network overhead for DCF mechanism when the network density is low. However, the network throughput of DCF method begins to decrease as more clients compete for the channel. Our proposed algorithms still keep high throughput in high density deployment. Obviously, the optimized CCA algorithm can achieve higher throughput compared with the other algorithms in high density network, which is due to the priority scheduling policy performing effectively.

5.3. Simulation in High Density Network Deployment. We also evaluate our proposed algorithms and TMCA algorithm in more complex and higher density networks; the scenarios are shown in Figure 8. 
TABLE 2: The relationship between effective bit rates and SINRs in IEEE 802.11 Standard.

\begin{tabular}{lcccccccc}
\hline$\gamma(\mathrm{dB})$ & $6-7.8$ & $7.8-9$ & $9-10.8$ & $10.8-17$ & $17-18.8$ & $18.8-24$ & $24-24.6$ & $>24.6$ \\
$C_{r}(\mathrm{Mbps})$ & 6 & 9 & 12 & 18 & 24 & 36 & 48 \\
\hline
\end{tabular}

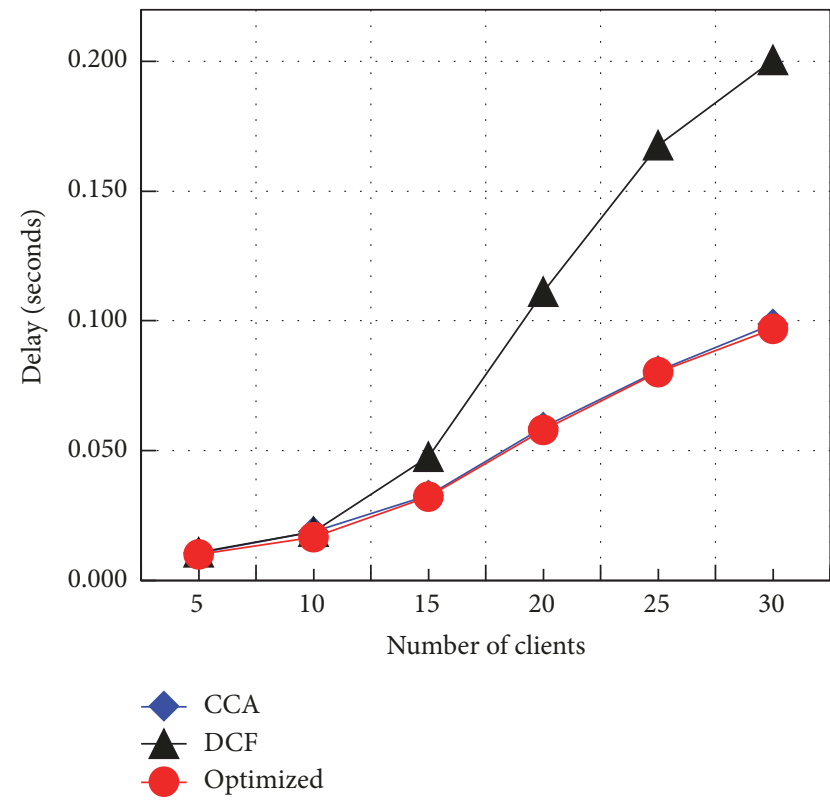

(a) The transmission delay in tight coupling deployment

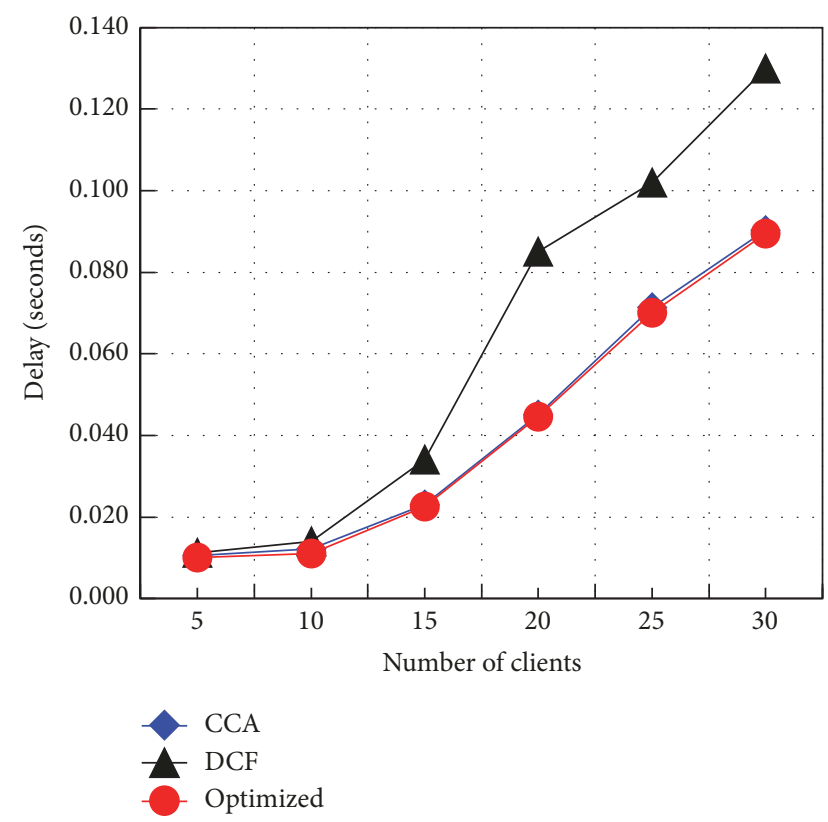

(b) The transmission delay in loose coupling deployment

FIgURE 5: Transmission delay under various network densities.

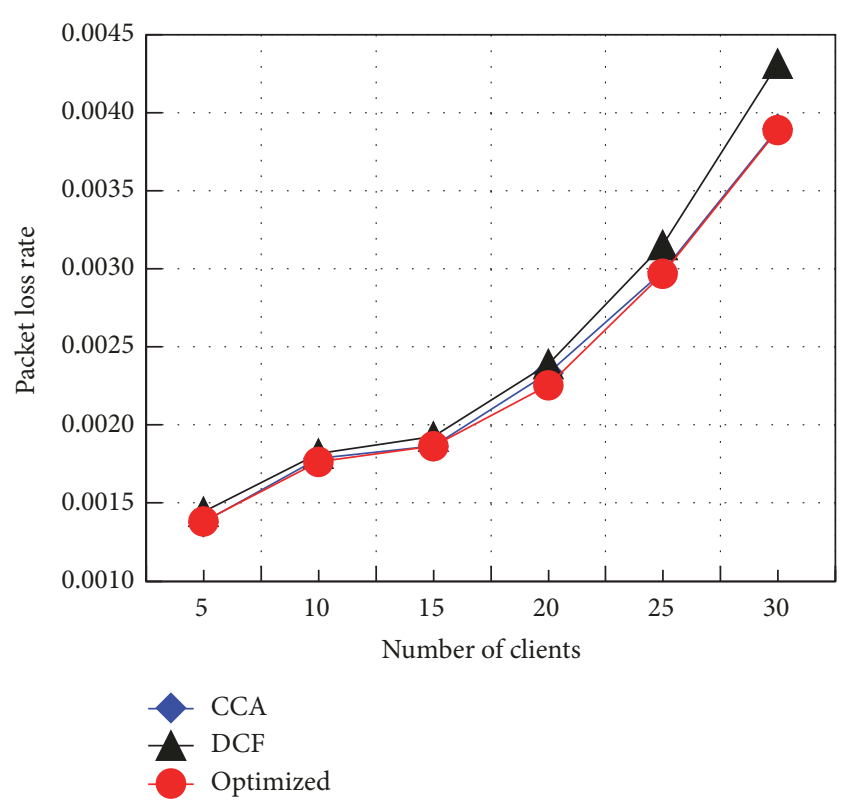

(a) The packet loss rate in tight coupling deployment

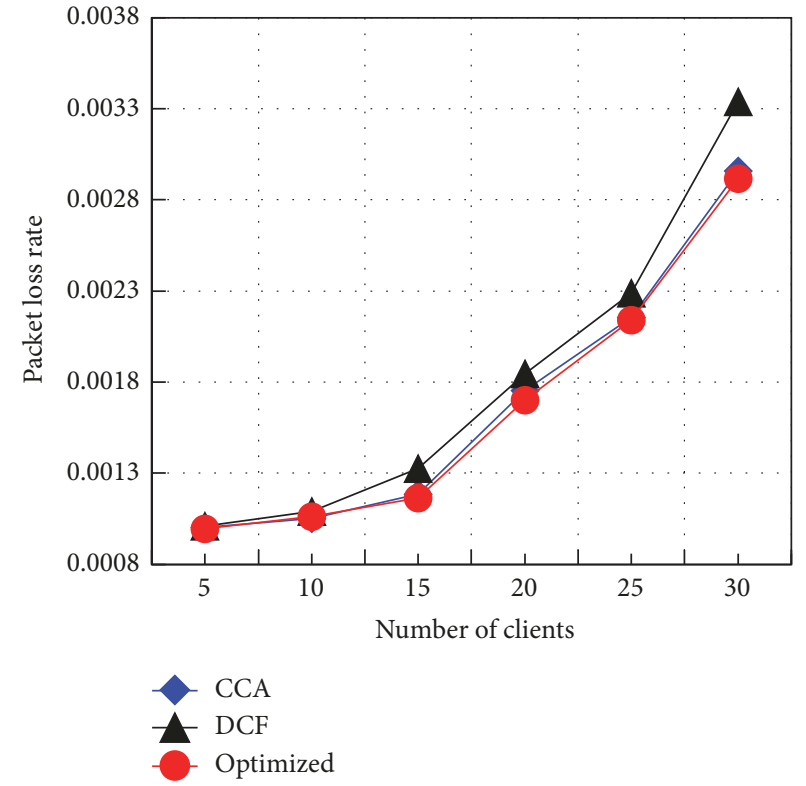

(b) The packet loss rate in loose coupling deployment

Figure 6: Packet loss rate under various network densities.

5.3.1. Transmission Delay. Figure 9 shows the transmission delay of our proposed algorithms and TMCA algorithm under the topology depicted in Figures 6(a) and 6(b). The CCA and optimized CCA algorithms perform better and they can reduce transmission delay by $15 \%$ compared with the TMCA algorithm. The TMCA algorithm performs badly in high density deployment due to its binary back-off algorithm. Nevertheless, our proposed algorithms can operate the channel access coordinately among APs and thus result in bounded access delay. 


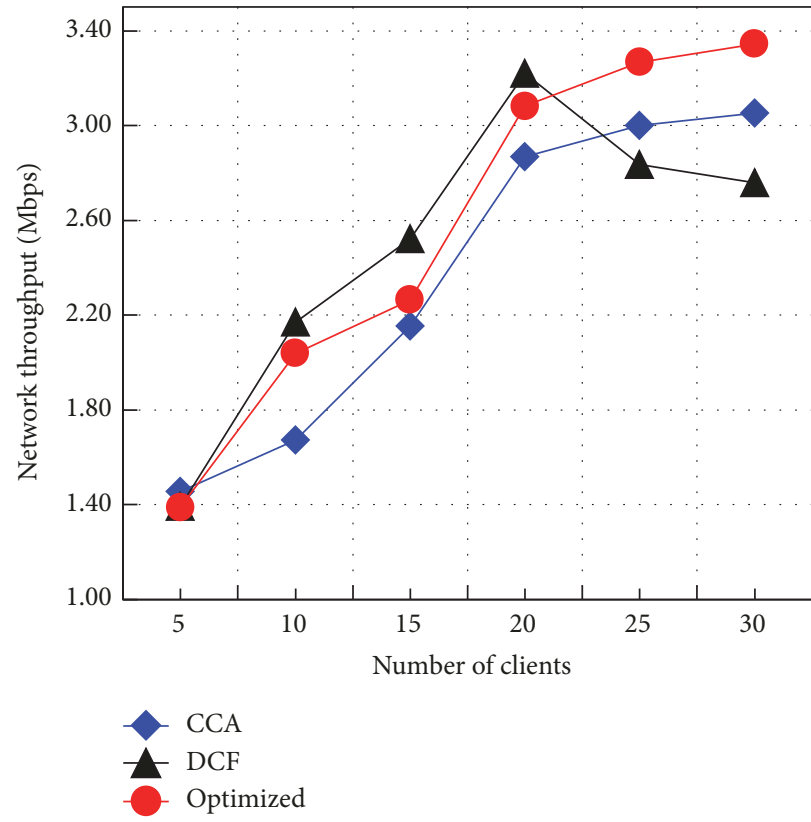

(a) The average network throughput in tight coupling deployment

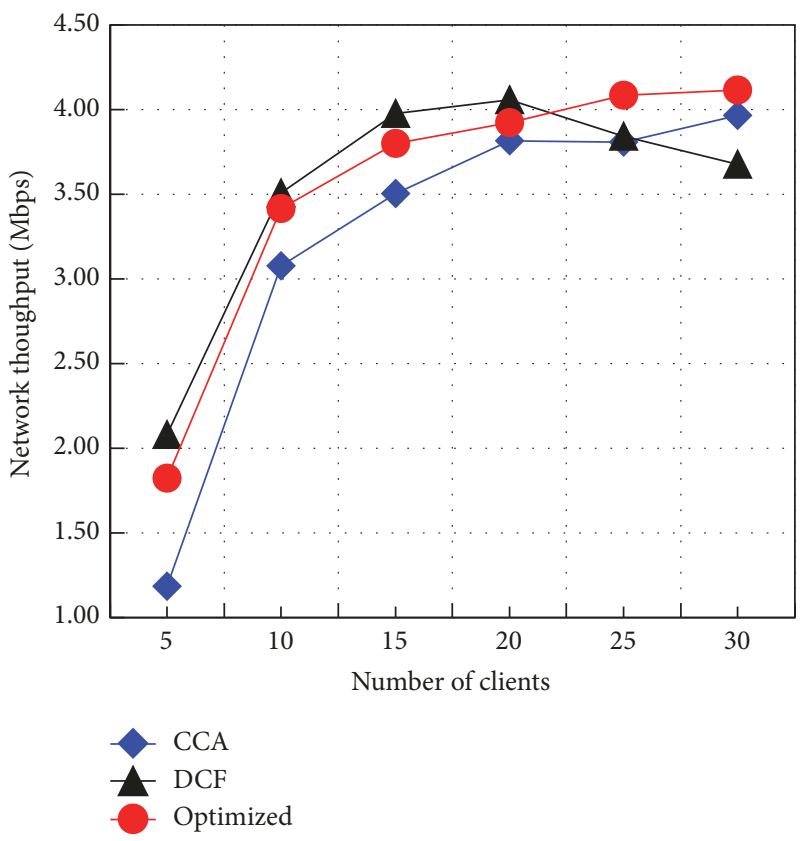

(b) The average network throughput in loose coupling deployment

FIGURE 7: Average network throughput under various network densities.

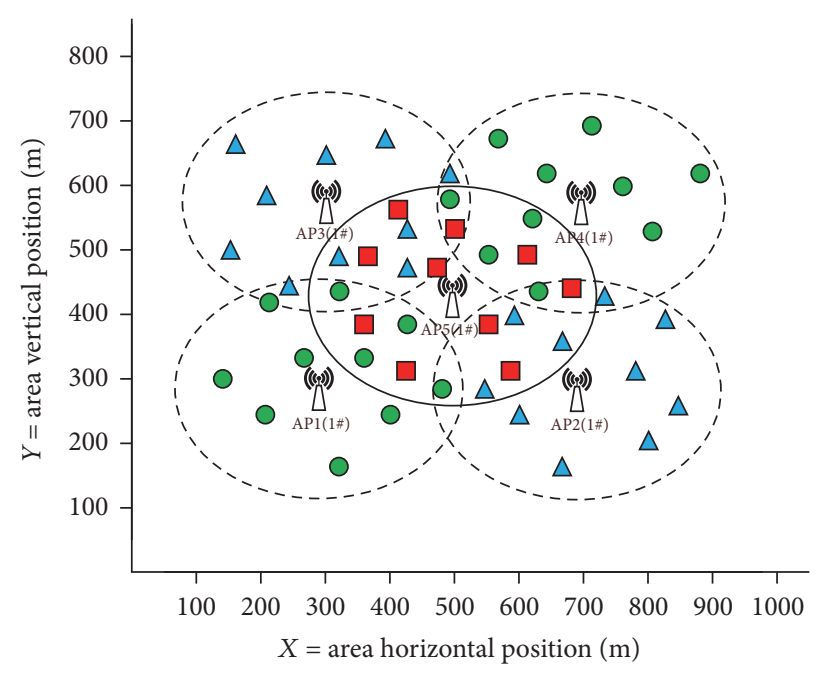

(a) 5 APs' scenario

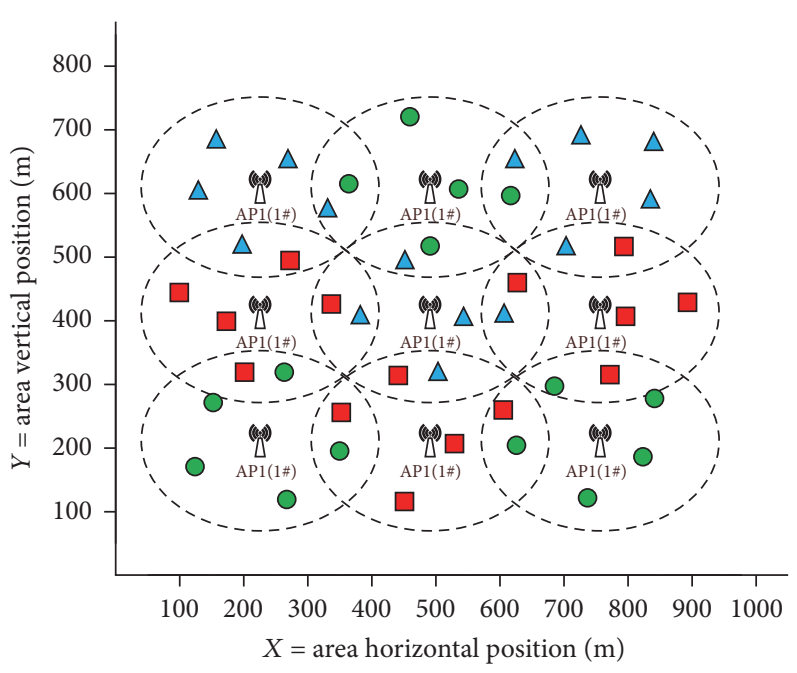

(b) 9 APs' scenario

FIGURE 8: High density network deployment with 5 and 9 APs.

5.3.2. Packet Loss Rate. Figure 10 shows the packet loss rate for 5 and 9 APs' deployment under various client densities. Obviously, our proposed CCA and optimized CCA algorithms can achieve lower packet loss rate compared with TMCA algorithm. The TMCA algorithm only has three nonoverlapped channels available, which can cause severe cochannel interference when more than three APs are deployed in the same overlap area. However, our proposed algorithms can coordinate AP operating to reduce collisions and packet loss rate.
5.3.3. Network Throughput. The TMCA, CCA, and optimized CCA algorithms are also evaluated in terms of the normalized throughput, which is computed by

$$
X_{\text {norm }}=\frac{X-X_{\min }}{X_{\max }-X_{\min }},
$$

where $X$ is the measurement data and $X_{\max }$ and $X_{\min }$ are the maximum and minimum of the measurement data, respectively. Figure 11 shows the simulation results for the number of clients for each AP increasing from 5 to 30 . The 


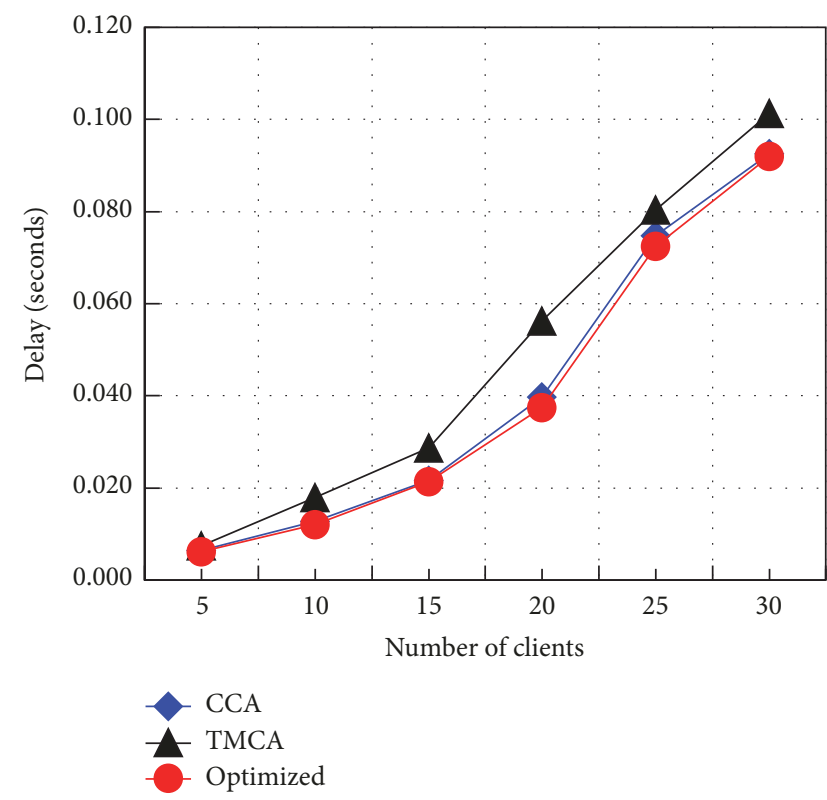

(a) The delay in the topology with 5 APs

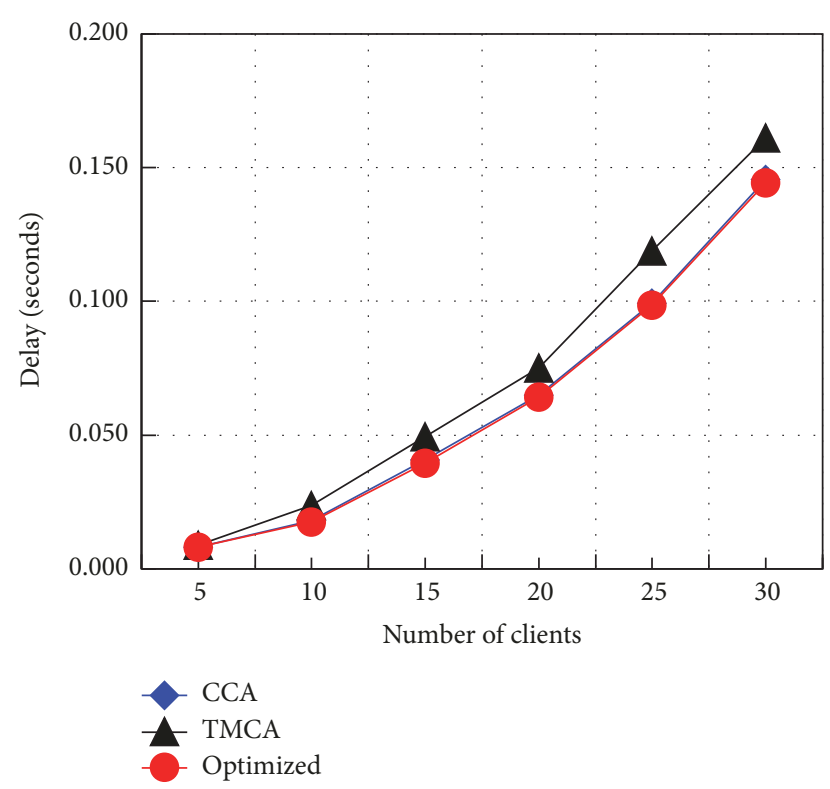

(b) The delay in the topology with 9 APs

FIgURE 9: Transmission delay in 5 and 9 APs' deployment under various network densities.

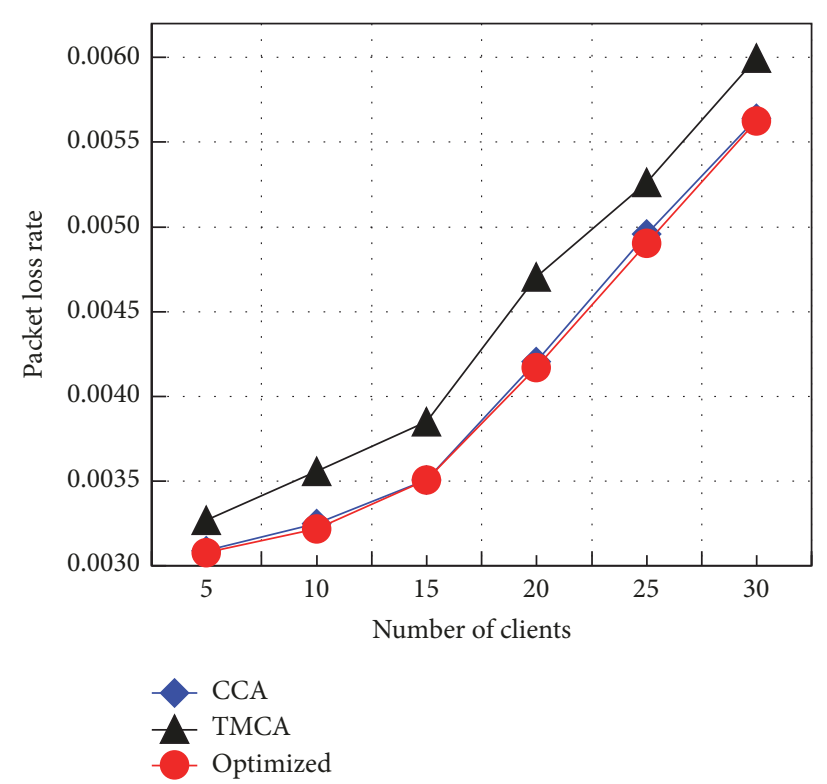

(a) The packet loss rate in the topology with 5 APs

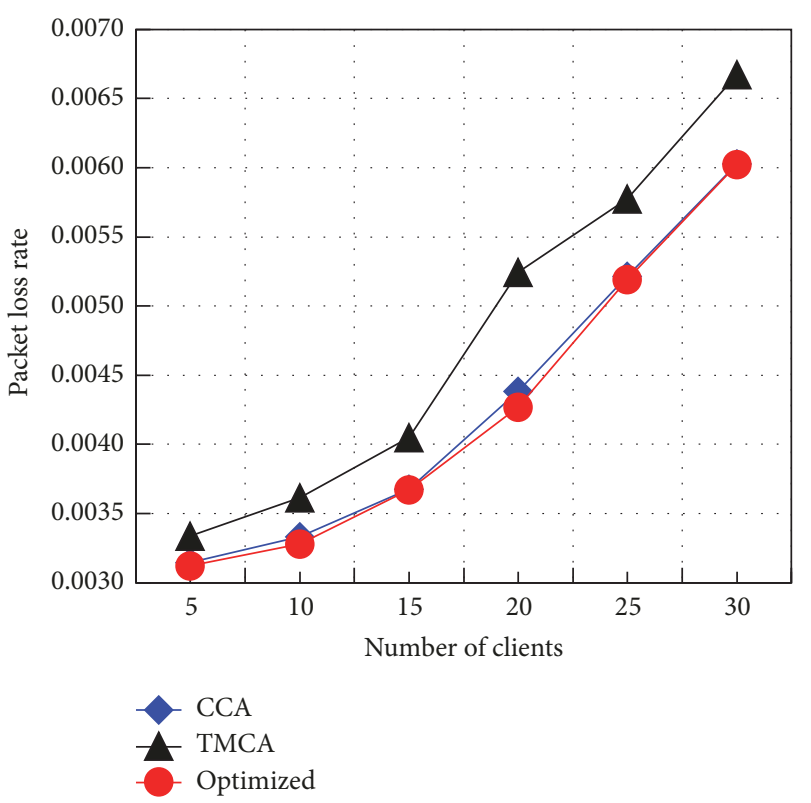

(b) The packet loss rate in the topology with 9 APs

Figure 10: Packet loss rate in 5 and 9 APs' deployment under various network densities.

throughput of TMCA algorithm is slightly higher than that of our proposed algorithms when the number of clients is less than 20. But as the number of clients increases, the throughput of TMCA algorithm decreases while those of the CCA and optimized CCA algorithms always stay at a high level and they can achieve 10\% improvement compared with TMCA, which is due to more collision for TMCA algorithm only using three nonoverlapped channels. Meanwhile, the optimized CCA algorithm performs more impressively since it optimizes the scheduling strategy and reduces the polling number of those clients without data transmission, thus improving the throughput.

\section{Conclusions}

In this paper, we propose a channel assignment algorithm based on PCF mechanism for multiple APs cochannel 


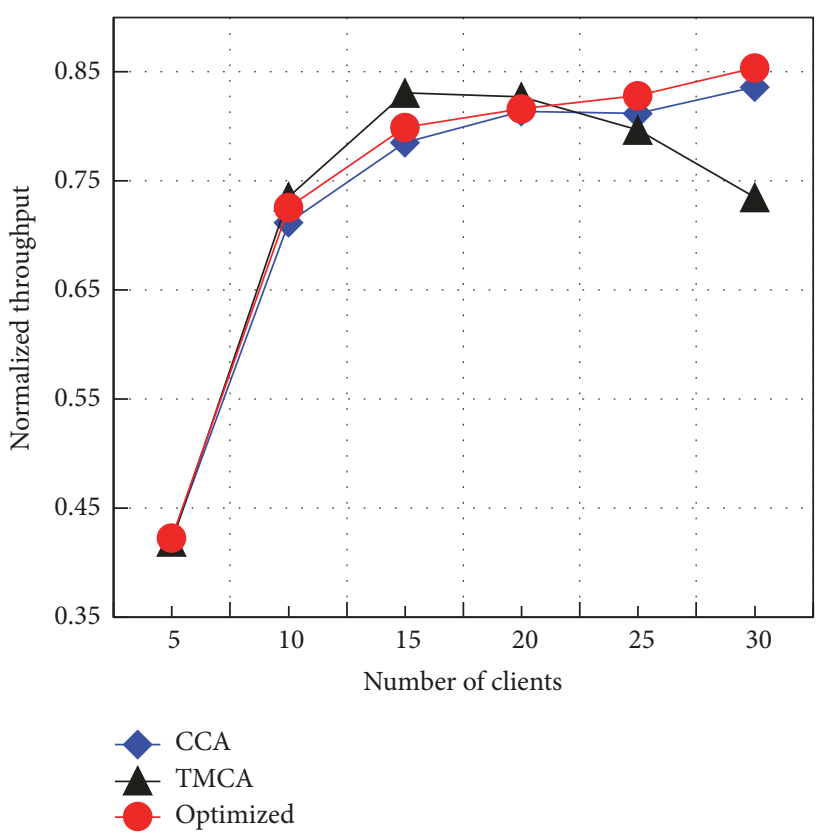

(a) The normalized throughput in the topology with 5 APs

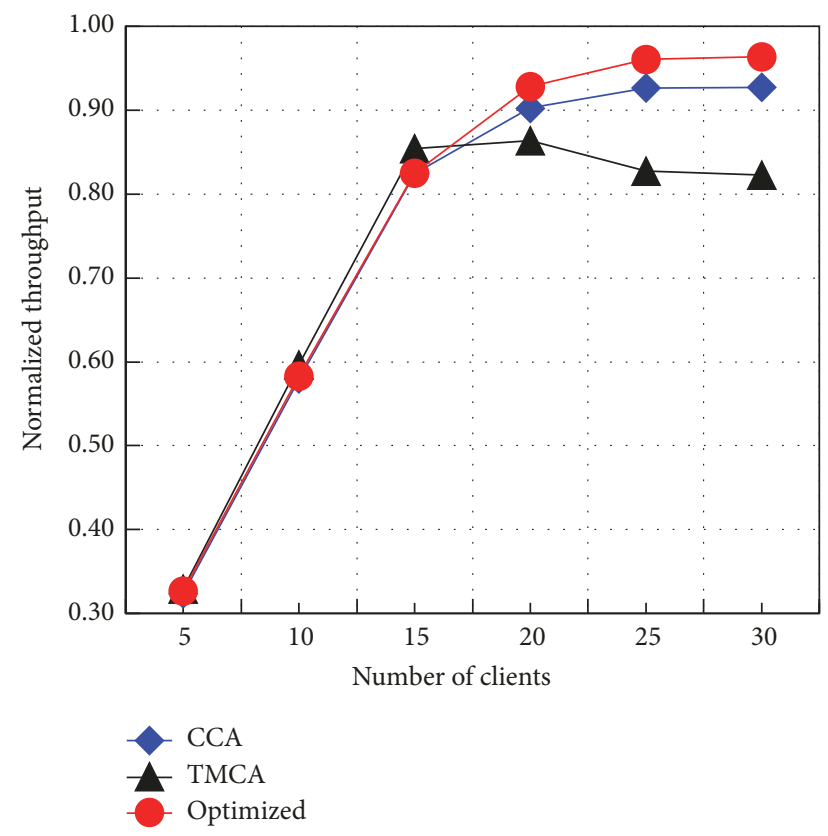

(b) The normalized throughput in the topology with 9 APs

FIgURE 11: Normalized throughput under various network densities in 5 and 9 APs' deployment.

deployment scenario. We present the network and interference model and formulate the problem of channel assignment into a vertex coloring problem. We propose the CCA algorithm which assigns the channel based on the vertex coloring approach, and then, by applying time slot reservation, make extra polls to improve the efficiency of channel assignment by applying time slot reservation. In addition, an optimized CCA algorithm also is proposed by classifying the clients and optimizing the polling list to reduce empty polls. Finally, we evaluate the proposed algorithms under different network densities, which shows that our proposed algorithm can obtain high throughput, low packet loss rate, and bounded access delay.

\section{Disclosure}

Figures 3, 4, and 8 and Table 1 are reprinted by permission from Springer Nature: Proceeding of the 6th Computer Science On-line Conference (CSOC2017) (A Novel CoChannel Deployment Algorithm Based on PCF in Multiple APs and High Density WLANs, Jianjun Lei and Jianhua Jiang) copyright 2017.

\section{Conflicts of Interest}

The authors declare that there are no conflicts of interest regarding the publication of this paper.

\section{Authors' Contributions}

Fengjun Shang contributed to presenting the priority channel access strategy and guiding the evaluating experiments for the optimized CCA mechanism.

\section{Acknowledgments}

This work was supported by the National Science Foundation of China (61672004), the Program for Innovation Team Building at Institutions of Higher Education in Chongqing (CXTDX201601021), and Scientific and Technological Research Program of Chongqing Municipal Education Commission (KJ1500439). This paper was presented in part at Springer CSOC2017. The authors would like to thank the anonymous CSOC2017 reviewers for their helpful comments on an earlier version of this work.

\section{References}

[1] S. Fiehe, J. Riihijvi, and P. Mahonen, "Experimental Study on Performance of IEEE 802.11n and Impact of Interferers on the 2.4 GHz ISM Band," in ACM IWCMC, pp. 47-51, 2010.

[2] D. Gong and Y. Yang, "AP Association in 802.11n WLANs with Heterogeneous Clients," in Proceedings of the IEEE Conference on Computer Communications (INFOCOM '12), pp. 1440-1448, March 2012.

[3] T. Cuzanauskas and A. Anskaitis, "Multi-polling game for IEEE 802.11 networks," in Proceedings of the 3rd IEEE Workshop on Advances in Information, Electronic and Electrical Engineering (AIEEE '15), November 2015.

[4] D. Zhao, M. Zhu, and M. Xu, "Leveraging SDN and OpenFlow to mitigate interference in enterprise WLAN," Journal of Networks, vol. 9, no. 6, pp. 1526-1533, 2014.

[5] J. Lei and J. Jiang, "A Novel Co-channel Deployment Algorithm Based on PCF in Multiple APs and High Density WLANs," in Proceedings of the the 6th Computer Science On-line Conference (CSOC '17), vol. 575, pp. 126-135, 2017.

[6] M. Achanta, Method and apparatus for least congested channel scan for wireless access points. USA, 20060072602, 2006. 
[7] H. Zhang, H. Ji, and W. Ge, "Channel assignment with fairness for multi-AP WLAN based on distributed coordination function," in Proceedings of the 2011 IEEE Wireless Communications and Networking Conference (WCNC '11), pp. 392-397, March 2011.

[8] Y. Liu, W. Wu, B. Wang, T. He, S. Yi, and Y. Xia, "Measurementbased channel management in WLANs," in Proceedings of the IEEE Wireless Communications and Networking Conference 2010 (WCNC '10), pp. 1-6, April 2010.

[9] C.-C. Hsu, Y.-A. Liang, J. L. G. Gomez, C.-F. Chou, and C.J. Lin, "Distributed flexible channel assignment in WLANs," in Proceedings of the 2013 IEEE Wireless Communications and Networking Conference (WCNC '13), pp. 493-498, April 2013.

[10] B. Kauffmann, F. Baccelli, A. Chaintreau, V. Mhatre, K. Papagiannaki, and C. Diot, "Measurement-based self organization of interfering 802.11 wireless access networks," in Proceedings of the IEEE INFOCOM 2007: 26th IEEE International Conference on Computer Communications, pp. 1451-1459, May 2007.

[11] H. Zhang, H. Ji, and W. Ge, "Channel Assignment with Fairness for multi-AP WLAN based on Distributed Coordination," in Proceedings of the 2011 IEEE Wireless Communications and Networking Conference (WCNC '11), pp. 392-397, March 2011.

[12] D. Xu, Z. Feng, Y. Li, and P. Zhang, "Fair channel allocation and power control for uplink and downlink cognitive radio networks," in Proceedings of the 2011 IEEE GLOBECOM Workshops (GC Wkshps '11), pp. 591-596, December 2011.

[13] Y. Wu, Y. Sun, Y. Ji, J. Mao, and Y. Liu, "A joint channel allocation and power control scheme for interference mitigation in highdensity WLANs," in Proceedings of the 15th IEEE International Conference on Communication Technology (ICCT '13), pp. 98103, November 2013

[14] D. Gong, Y. Yang, and H. Li, "High-throughput collision-free client polling in multi-AP WLANs," in Proceedings of the 54th Annual IEEE Global Telecommunications Conference: "Energizing Global Communications” (GLOBECOM '11), December 2011.

[15] Y. Bejerano and R. S. Bhatia, "MiFi: A framework for fairness and QoS assurance for current IEEE 802.11 networks with multiple access points," IEEE/ACM Transactions on Networking, vol. 14, no. 4, pp. 849-862, 2006.

[16] H. Nishimura, R. Fujiwara, M. Miyazaki, and M. Katagishi, "Access point cooperation for collision-free polling between distributed real-time networks," in Proceedings of the 26th IEEE Annual International Symposium on Personal, Indoor, and Mobile Radio Communications, PIMRC 2015, pp. 1057-1061, China, September 2015.

[17] S. Gualandi and F. Malucelli, "Exact solution of graph coloring problems via constraint programming and column generation," INFORMS Journal on Computing, vol. 24, no. 1, pp. 81-100, 2012.

[18] Q. Wu and J. K. Hao, "Coloring large graphs based on independent set extraction," Computers and Operations Research, vol. 39, pp. 283-290, 2012.

[19] L.-Y. Bao, D.-F. Zhao, and Y.-F. Zhao, "An efficient polling scheme for wireless LANs," in Proceedings of the 2010 International Conference on Computer Design and Applications, ICCDA 2010, pp. V4540-V4544, China, June 2010.

[20] Q. Liu, D. Zhao, and H. Ding, "An improved polling scheme for PCF MAC protocol," in Proceedings of the 7th International Conference on Wireless Communications, Networking and Mobile Computing (WiCOM '11), pp. 407-411, September 2011.

[21] J.-Y. Yeh and C. Chen, "Support of multimedia services with the IEEE 802.11 MAC protocol," IEEE International Conference on Communications, vol. 1, pp. 600-604, 2002.
[22] D. Gong, M. Zhao, and Y. Yang, "Channel assignment in multirate 802.11n WLANs," in Proceedings of the 2013 IEEE Wireless Communications and Networking Conference (WCNC '13), pp. 392-397, April 2013.

[23] Y. Cui, W. Li, and X. Cheng, "Partially overlapping channel assignment based on "node orthogonality" for 802.11 wireless networks," in Proceedings of the IEEE INFOCOM 2011, pp. 361365, April 2011. 


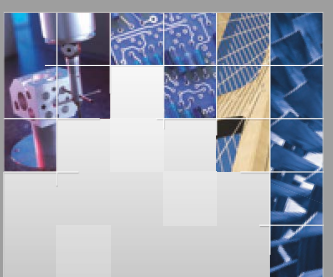

\section{Enfincering}
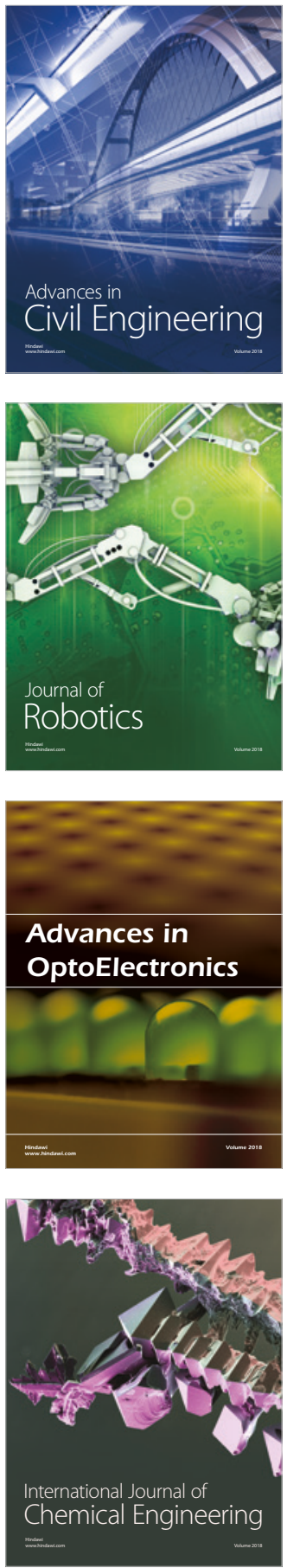

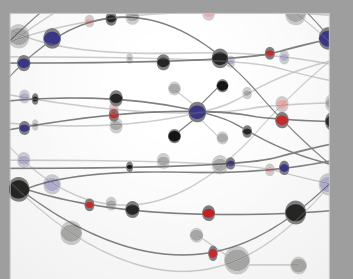

\section{Rotating \\ Machinery}

The Scientific World Journal

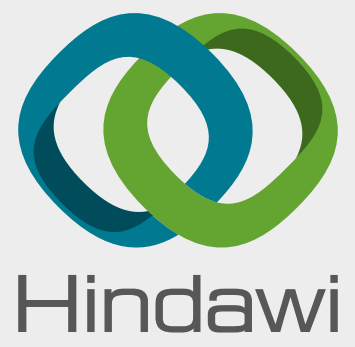

Submit your manuscripts at

www.hindawi.com
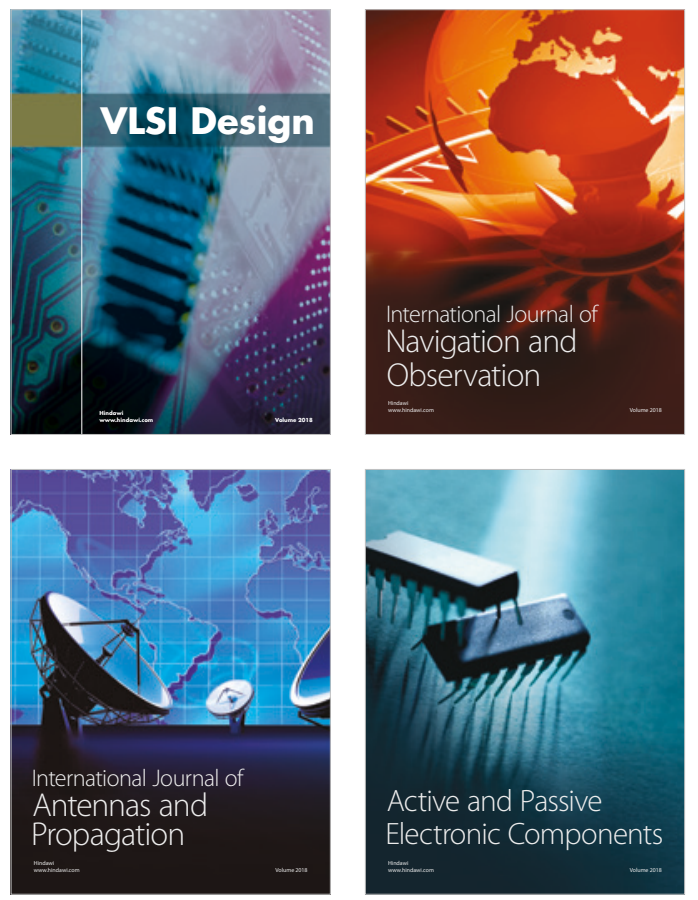
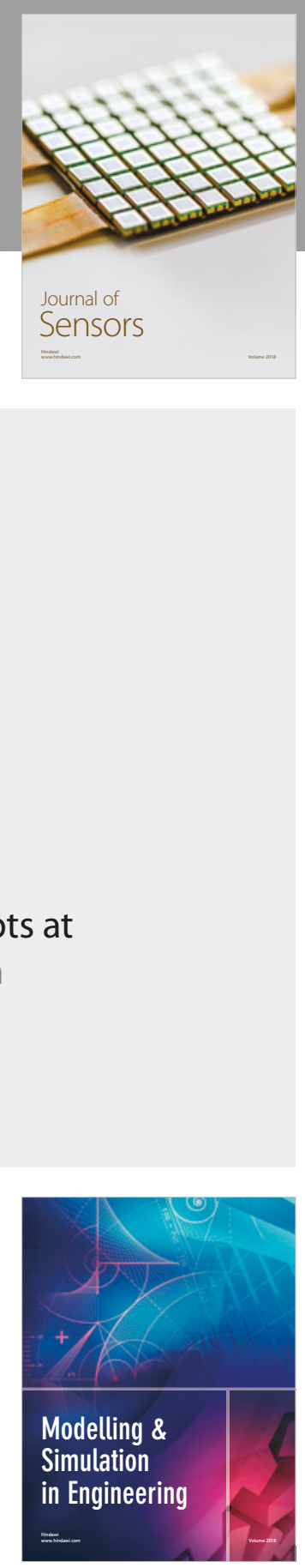

\section{Advances \\ Multimedia}
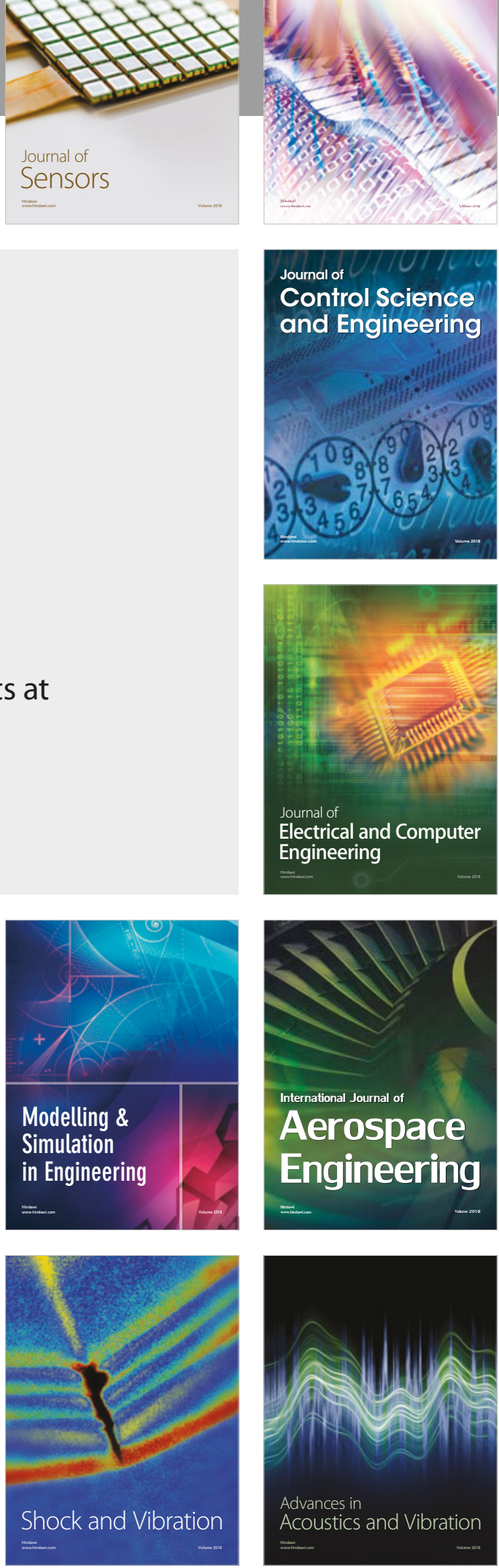